\title{
DAMAGE DIAGNOSIS IN BEAM-LIKE STRUCTURES BY ARTIFICIAL NEURAL NETWORKS
}

\author{
Kamil AYDIN ${ }^{\mathrm{a}}$, Ozgur KISI ${ }^{\mathrm{b}}$ \\ ${ }^{a}$ Department of Civil Engineering, Faculty of Engineering, Erciyes University, 38039 Kayseri, Turkey \\ ${ }^{b}$ Department of Civil Engineering, Canik Basari University, Ilkadim, Samsun, Turkey
}

Received 30 Sep 2012; accepted 12 Nov 2012

\begin{abstract}
Applicability of artificial neural networks is examined in determining the natural frequencies of intact beams and crack parameters of damaged beams. Multi-layer perceptron (MLP) and radial basis neural networks (RBNN) are utilized for training and validation of input data. In the first part of the study, the first four frequencies of free vibration are predicted based on beam properties by the networks. Showing the effectiveness of the neural networks in predicting the vibrational frequencies, the second part of the study is carried out. At this stage of the inverse problem, the frequencies and mode shape rotation deviations in addition to beam properties are used as input to the networks to determine the crack parameters. Different hidden nodes, epochs and spread values are tried to find the optimal neural networks that give the lowest error estimates. In both parts of the study, the RBNN model performs better. The robustness of the network models in the presence of noise is also shown. It is shown that the optimal MLP network predicts the crack parameters slightly better in the presence of noise. As a conclusion, the trained RBNN model can be used in health monitoring of beam-like structures as a crack identification algorithm.
\end{abstract}

Keywords: artificial neural networks, MLP, RBNN, beam, crack.

\section{Introduction}

Engineering structures accumulate damage during their service life. The occurrence of damage in civil, mechanical and aerospace engineering structures might be unavoidable. However, the earliest possible detection of damage is crucial in order to prevent catastrophic, economic and human loss. Damage detection methods can be ordinarily classified as local and global damage identification techniques (Doebling et al. 1996). Local damage detection methods are based on visual inspection, stress waves, ultrasonic waves, X-ray, acoustics or radiography. These methods are restricted to local examination in limited areas and require a prior knowledge of the vicinity of damage (Yan et al. 2007). When applied to large structures, these methods are very time consuming and costly (Carden, Fanning 2004). To overcome these difficulties, global damage identification methods have been developed (Fan, Qiao 2011). Global methods referred to as vibration-based damage detection techniques assess the condition of the entire structure at once. These methods are based on the basic principle that damages emerging in a structure alters both its physical properties (e.g. mass, stiffness and damping) and dynamic properties (e.g. frequency response function, natural frequencies, damping ratios and mode shapes) (Shi et al. 2000a, 2002; Gawronski, Sawicki 2000; Kawiecki 2001; Abdo, Hori 2002; Sampaio et al. 2003). By analysing the dynamic characteristics of a structure, hence, any damage can be identified, including its location and degree. Consequently, intensive research has been carried out in the field of vibration-based damage identification over the last three decades. A broad range of techniques, algorithms and methods utilizing natural frequencies (Gudmundson 1982; Kasper et al. 2008; Nandwana, Maiti 1997), mode shapes (Shi et al. 2000b; Lee et al. 2005; Abdo, Hori 2002), mode shape curvature (Pandey et al. 1991; Abdel Wahab, De Roeck 1999; Ratcliffe 2000), modal flexibility (Pandey, Biswas 1994; Wu, Law 2004, 2005), etc. are developed to solve challenging problems encountered in various structures, from basic structural components (e.g. beams and plates) to complex structural systems (e.g. buildings and bridges).

Vibration-based damage identification is an inverse problem of predicting crack location and severity from the modal information of structures. Problems exist with dynamic-based inverse procedures. The inverse system identification techniques deteriorate in the presence of noise. Also, a unique solution often does not exist for an inverse problem, particularly when the relationships between damage properties and dynamic changes in structures

Corresponding author: Kamil Aydin

E-mail:kaydin@erciyes.edu.tr 
are very complex and only limited data are available (Bakhary et al. 2007). Vibration-based damage detection is overall a pattern recognition problem, where alterations in the vibrational characteristics of a structure are attributed to certain properties of damage. Therefore, a different approach is required to overcome these critical issues of traditional damage detection methods. Artificial neural networks (ANNs) are such one method. ANNs are reliable damage identification methods due to their capabilities of pattern recognition and classification. They have the noise filtering capabilities that make them more robust in the presence of measurement noise and other uncertainties. Besides, once ANNs are properly established, damage identification is relatively fast and mathematical models do not need to be constructed (Bakhary 2008).

ANN is a mathematical model of biological neural system and theoretical mind. The basic concept of utilizing ANN for damage identification is to build a model to provide a relationship between the measured parameters of structures such as modal information, frequency response functions, time domain data, etc., and structural parameters via a training process. Once the relationship is set up, the trained ANN model is capable of detecting damage from the measured quantities. The first successful application of ANN using dynamic parameters of civil structures in the field of damage identification was carried out by Wu et al. (1992). Since then, the ANN - based damage identification techniques have been used for various structures such as beams (Marwala, Hunt 1999), frame structures (Nikolakopoulos et al. 1997), plates (Liu et al. 2002), trusses (Yun, Bahng 2000) and composite frames (Zapico et al. 2003). The following presents a review of recent work performed for structural beam elements only, as the study herein considers the ANN-based damage detection in beam-type structures.

Both global (changes in natural frequencies) and local (changes in strain mode shapes) dynamic-based analysis data have been used as input in ANNs for location and severity prediction of crack damage in beamlike structures. Natural frequencies computed for various crack locations and depth using a fracture mechanics based crack model were used to train a neural network to identify both the crack location and height (Suresh et al. 2004). A modular neural network architecture with two widely used neural networks, i.e. the multi-layer perceptron (MLP) network and the radial basis neural networks (RBNN), was employed for the comparative study. Sahin and Shenoi (2003) developed a damage detection algorithm using a combination of global (natural frequencies) and local (curvature mode shapes) data to identify the location and depth of cracks in beam structures. The dynamic properties of intact and cracked cantilever steel beams for the first three natural modes were obtained from a finite element program. The formed ANN architecture was tested against noisy data and experimental damage cases. The performance of ANN is compared to that support vector machine (SVM) by Liu and Meng
(2005) in crack detection using a few natural frequencies of supported beams. The analysis results showed that SVM is a little better in locating and sizing the cracks. The inverse problem of finding the crack location and depth in a cantilever Euler-Bernoulli beam and a rotating beam was studied by Rosales et al. (2009). Two approaches, power series technique (PST) and ANNs, were presented to solve the inverse problem. It was concluded that PST is straightforward and identifies the crack with small errors. However, the simplicity of this approach limits its use. Though the ANN yields larger errors on the average, it is capable of handling more complex models. Detection of crack in a cantilever beam through ANN was also studied by Das and Parhi (2009). The first three natural frequencies and mode shapes were used as input parameters to the ANN. The output parameters of the network were the relative crack depth and location. Several training patterns were derived in the network. The robustness of the developed network was verified using the experimental results. The use of ANN for crack prediction in curvilinear beam was recently studied by Saeed and George (2011).Vibration characteristics of intact and damaged beams were numerically computed from finite element method. Natural frequencies and frequency response functions were fed as input into ANN, which then estimated the size of crack and its location.

The literature that has contributed to the vibrationbased damage detection using ANN over the past two decades can be categorized into four groups according to the input they employ: ANNs-based methods using modal parameters, methods using both modal parameters and their derivatives, methods using frequency response functions and methods using time domain data. In those procedures utilizing modal parameters only, a two-stage identification technique is performed due to complex computation involved in neural network and non-uniqueness of the inverse problem. The idea of employing two-stage identification is to reduce the number of variables. Location of crack is predicted in the first stage of these methods. The crack severity is then determined in the second stage. In addition to modal parameters, the second group of methods uses their derivatives to avoid a multi-stage computation. In that case, however, a large number of data needs to be made available since training a neural network with large data provides a significant amount of redundancy and a better learning algorithm. It can also avoid the problem of over fitting, which can be defined as the inability of a network to perform better when unseen test data are input to the network. The ANNs trained with frequency response functions ortime domain data generally require excessive number of input parameters, which may as a consequence result in large and complex models. In order to circumvent sophisticated neural network models, data reduction techniques such as principal component analysis or singular value decomposition are carried out to reduce the computed input data sets. 
Most ANN models are limited to example structures with a small number of degrees of freedom or small number of large structural elements. Since large elements are not sensitive to a small crack, severe damage scenarios are usually assumed to successfully apply ANNs to damage identification. Most studies, therefore, consider a single type of structural beam element. Also, the material and geometrical properties of beam-like structures are never includedin the neural networks.

The study herein investigates the feasibility of ANNs as a crack identification technique. The newness of the study is: 1) to incorporate the material and geometrical characteristics of beam elements in the neural network; 2) to consider a variety of beam types in a single neural network model; and 3) to utilize mode shape rotation deviation curve for the first time in training the ANNs. Euler-Bernoulli theory is assumed to model the beam elements. Two popularly used neural network models, namely MLP and RBNN networks, are used for the forward and inverse problems. It is reported in the literature that ANNs can learn about the behaviour of undamaged structures. Due to this and a large number of parameters involved in network architecture, the capabilities of the two ANNs are first examined in the forward problem. Having shown the robustness of the ANN procedures, the inverse problem of identifying the crack parameters, crack location and crack depth, are next studied. At this stage, the beam material and geometrical characteristics, support types of beam structures, the natural frequencies and mode shape rotation deviation values of the first four modes are used as input to train the network models. The outcomes of the trained ANNs are the relative crack location and relative crack depth. Despite a very large number of input parameters, both the forward problem and inverse problem of identification are carried out at a single-stage and no reduction analysis of network parameters is performed due to proper arrangement of network models.

\section{Theoretical analysis of beam}

In this study, flexural vibration of beams with rectangular cross section having a transverse crack extending uniformly along the width of the beam is considered. The beam with two ends supported by transverse and rotational springs is analysed as, by changing the stiffnesses of the springs, any support conditions of interest can be obtained. A beam of length $L$ with $n$ open cracks located at distance of $x_{1}, x_{2}, \ldots x_{n}$ such that $0<x_{1}<x_{2}<\ldots x_{n}<L$ is shown in Figure 1. The entire beam is now divided into $n+1$ intact segments. The cracks are modelled by massless rotational springs (Li 2000). One major assumption of this modelling is that the cracks are always open during the beam vibration and, hence, the sophisticated nonlinear dynamics of cracks that open and close during the motion are not considered. The $x$-coordinate is along the beam length and $y$ is along the beam height. $v$ is the transverse displacement and $\phi$ is the slope of the beam. The additional flexibility induced by the presence of $i^{\text {th }}$ crack is defined by the local compliance $C_{i}$, which causes a jump in the slope of the beam at that location, as shown in Figure $1 \mathrm{~b}$.

The governing differential equation of flexural vibration of intact Euler-Bernoulli beam is:

$$
\frac{\partial^{4} v(x, t)}{\partial x^{4}}+\frac{\bar{m}}{K_{b}} \frac{\partial^{2} v(x, t)}{\partial t^{2}}=0
$$

where: $K_{\mathrm{b}}=E I$ is the flexural stiffness; $E$ is the Young's modulus of elasticity; $I$ is the area moment of inertia; $\bar{m}=\rho A$ is the mass per unit length; $\rho$ is the mass density; and $A$ is the area of beam cross-section.

Assuming the variation of transverse deflection of the beam at any location to be:

$$
v(x, t)=X(x) e^{i \omega t}
$$

Eqn (1) becomes:

$$
\frac{d^{4} X(x)}{d x^{4}}-\frac{\bar{m} \omega^{2}}{K_{b}} X(x)=0
$$

In Eqn (2), $i-\sqrt{-1}, \omega$ and $X(x)$ are the circular frequency and mode-shape function of the beam respectively.
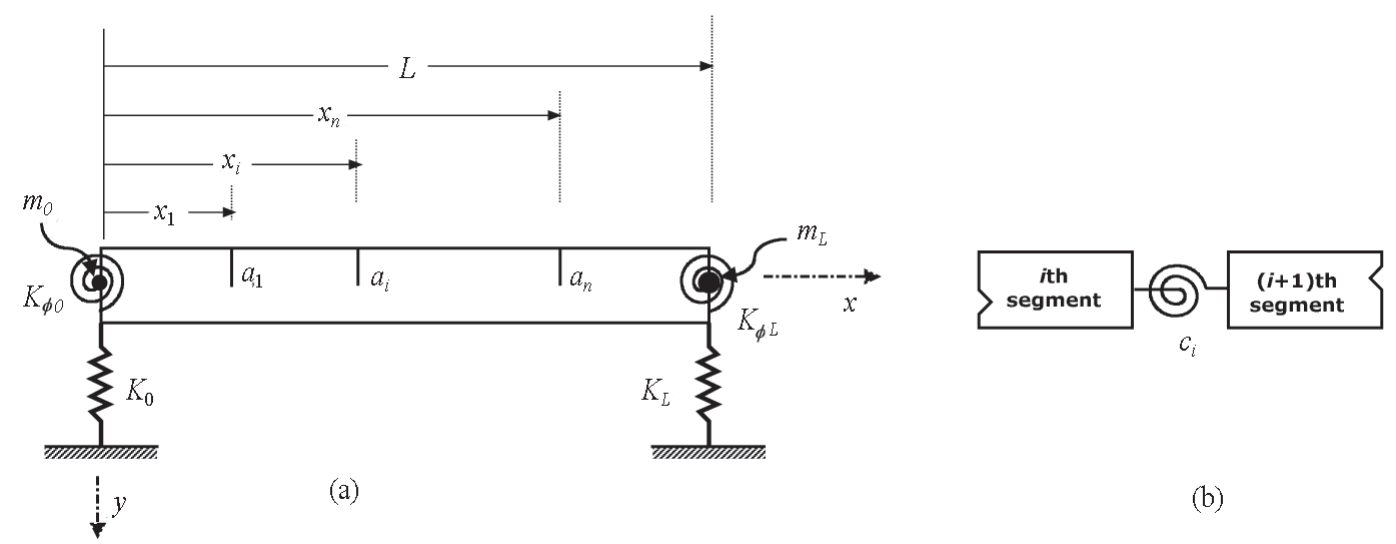

Fig. 1. Euler-Bernoulli beam having $n$ cracks (a), crack modelling as rotational spring (b) 
The general form of the solution for Eqn (3) is given by:

$$
X(x)=D_{1} S_{1}(x)+D_{2} S_{2}(x)+D_{3} S_{3}(x)+D_{4} S_{4}(x),
$$

where $D_{j}(j=1,2,3,4)$ 's are constants to be determined from the boundary conditions and $S_{j}$ 's are the linearly independent solutions given by:

$$
\begin{aligned}
& S_{1}(x)=\sin (k x) \\
& S_{2}(x)=\cos (k x), \\
& S_{3}(x)=\sinh (k x) \\
& S_{4}(x)=\cosh (k x)
\end{aligned}
$$

where: $k=\sqrt[4]{\bar{m} \omega^{2} L^{4} / K_{b}}$. To simplify the analysis of vibration of the Euler-Bernoulli beam, the linearly independent fundamental solutions denoted by $\bar{S}_{j}(x)(j=1,2,3,4)$ are constructed. This is done by satisfying the normalization condition at the origin of axes (see for details Li 2001). Consequently, the following are obtained:

$$
\begin{gathered}
\bar{S}_{1}(x)=\frac{1}{2} \cos (k x)+\frac{1}{2} \cosh (k x) \\
\bar{S}_{2}(x)=\frac{1}{2 k} \sin (k x)+\frac{1}{2 k} \sinh (k x) \\
\bar{S}_{3}(x)=-\frac{1}{2 k^{2}} \cos (k x)+\frac{1}{2 k^{2}} \cosh (k x) \\
\bar{S}_{4}(x)=-\frac{1}{2 k^{3}} \sin (k x)+\frac{1}{2 k^{3}} \sinh (k x)
\end{gathered}
$$

Using the above solutions for $\bar{S}_{j}$ 's, the mode-shape of the first segment $\left(0 \leq x<x_{1}\right)$ can be formulated as:

$$
\begin{gathered}
X_{1}(x)=X(0) \bar{S}_{1}(x)+\phi(0) \bar{S}_{2}(x)-\frac{M(0)}{K_{b}} \bar{S}_{3}(x)- \\
\frac{V(0)}{K_{b}} \bar{S}_{4}(x),
\end{gathered}
$$

where: $X(0), \phi(0), M(0)$ and $V(0)$ are the displacement, rotation, moment and shearing force of the beam at $x=0$ respectively. Only two of these parameters are unknown for any type of support conditions ( $\mathrm{Li} 2000$ ).

The compatibility conditions enforce the continuity of the force and displacement fields across the crack. For a crack located at section $x_{i}$, these conditions can be expressed as:

$$
\begin{gathered}
X_{i+1}\left(x_{i}\right)=X_{i}\left(x_{i}\right) \\
V_{i+1}\left(x_{i}\right)=V_{i}\left(x_{i}\right), \\
M_{i+1}\left(x_{i}\right)=M_{i}\left(x_{i}\right) \\
X_{i+1}^{\prime}\left(x_{i}\right)=X_{i}^{\prime}\left(x_{i}\right)+C_{i} X_{i}^{\prime \prime}\left(x_{i}\right)
\end{gathered}
$$

where $C_{i}$ is the compliance of the crack at the $i^{\text {th }}$ section. The expressions in Eqn (8) show the equalities of displacement, shear force, bending moment and rotation, respectively, at the common interface of the $i^{\text {th }}$ segment and the $(i+1)^{\text {th }}$ segment. $C_{i}$ can be expressed as:

$$
C_{i}=5.346 h f\left(a_{i} / h\right),
$$

where: $h$ is the height of the beam cross section; and $a_{i}$ is the depth of the $i^{\text {th }}$ crack. The function $f\left(a_{i} / h\right)$ is the dimensionless local flexibility computed from the strain energy function (Dimarogonas 1996) and given by:

$$
\begin{gathered}
f\left(a_{i} / h\right)=1.862\left(a_{i} / h\right)^{2}-3.95\left(a_{i} / h\right)^{3}+ \\
16.375\left(a_{i} / h\right)^{4}-37.226\left(a_{i} / h\right)^{5}+76.81\left(a_{i} / h\right)^{6}- \\
126\left(a_{i} / h\right)^{7}+172\left(a_{i} / h\right)^{8}-143.97\left(a_{i} / h\right)^{9}+ \\
66.56\left(a_{i} / h\right)^{10} .
\end{gathered}
$$

The mode-shape function for the section $\left(x_{i} \leq x<x_{i+1}\right)$ after the $i^{\text {th }}$ crack can be determined based on Eqns (7) and (8) as:

$$
X_{i+1}(x)=X_{i}(x)+C_{i} X_{i}^{\prime \prime}\left(x_{i}\right) \bar{S}_{2}\left(x-x_{i}\right) H\left(x-x_{i}\right),
$$

in which $H(\cdot)$ is the Heaviside function, which assumes 0 for $x<x_{i}$ and 1 for $x>x_{i}$. This equation is a recurrence formula of the mode-shape functions. Using this equation and the mode-shape function of the first segment, one can derive the mode-shape of the last segment (segment $n+1$ ) of the beam as:

$$
\begin{gathered}
X_{n+1}(x)=X_{1}(x)+\sum_{i=1}^{n} C_{i} X_{i}^{\prime \prime}\left(x_{i}\right) \bar{S}_{2}\left(x-x_{i}\right) H\left(x-x_{i}\right) . \\
i=1,2,3, \ldots n
\end{gathered} .
$$

The characteristic equation is readily established by imposing boundary conditions on the above equation. This is outlined for a cracked beam with two ends supported by springs and with end masses.

The beam shown in Figure 1a has a transverse spring of stiffness $K_{0}$, rotational spring of stiffness $K_{\phi 0}$ and concentrated mass of $m_{0}$ at the left end. The boundary conditions are then:

$$
\begin{aligned}
& V(0)=\left(K_{0}-m_{0} \omega^{2}\right) X(0) \text { and } \\
& M(0)=-K_{\phi 0} \phi(0) .
\end{aligned}
$$

Replacing the $V(0)$ and $M(0)$ in Eqn (7) by those in Eqn (13) gives the first segment of the beam as:

$$
\begin{gathered}
X_{1}(x)=\left[\bar{S}_{1}(x)-\frac{K_{0}-m_{0} \omega^{2}}{K_{s}} \bar{S}_{4}(x)\right] X(0)+ \\
{\left[\bar{S}_{2}(x)+\frac{K_{\phi 0}}{K_{b}} \bar{S}_{3}(x)\right] \phi(0) .}
\end{gathered}
$$

Eqn (14) can be rewritten in a compact form:

$$
X_{1}(x)=\bar{S}_{11}(x) X(0)+\bar{S}_{22}(x) \phi(0),
$$


where:

$$
\begin{aligned}
& \bar{S}_{11}(x)=\bar{S}_{1}(x)-K_{m 0} \bar{S}_{4}(x) \\
& \bar{S}_{22}(x)=\bar{S}_{2}(x)+\mu_{\phi 0} \bar{S}_{3}(x)
\end{aligned}
$$

with $K_{m 0}=\frac{K_{0}-m_{0} \omega^{2}}{K_{s}}$ and $\mu_{\phi 0}=\frac{K_{\phi 0}}{K_{b}}$.

The support conditions at the right end are:

$$
\begin{aligned}
& X_{n+1}^{\prime \prime}(L)+\mu_{\phi L} X_{n+1}^{\prime}(L)=0 \\
& X_{n+1}^{\prime \prime \prime}(L)-K_{m L} X_{n+1}(L)=0
\end{aligned}
$$

where:

$$
K_{m L}=\frac{K_{L}-m_{L} \omega^{2}}{K_{b}} \text { and } \mu_{\phi L}=\frac{K_{\phi L}}{K_{b}} \text {. }
$$

In this equation, $K_{L}$ and $K_{\phi L}$ are the stiffness of the transverse spring and the rotational spring, and $m_{L}$ is the concentrated mass at the right end support. Using Eqns (11), (15) and (17) leads to:

$$
\left[\begin{array}{c}
\bar{S}_{11}^{\prime \prime}(L)+\mu_{\phi L} \bar{S}_{11}^{\prime}(L)+ \\
\sum_{i=1}^{n} C_{i} \bar{S}_{11}^{\prime \prime}\left(x_{i}\right)\left\{\bar{S}_{2}^{\prime \prime}\left(L-x_{i}\right)+\mu_{\phi L} \bar{S}_{2}^{\prime}\left(L-x_{i}\right)\right\}+ \\
\sum_{i=1}^{n-1} C_{i} C_{i+1} \bar{S}_{11}^{\prime \prime}\left(x_{i}\right) \bar{S}_{2}^{\prime \prime}\left(x_{i+1}-x_{i}\right) \\
\left\{\bar{S}_{2}^{\prime \prime}\left(L-x_{i+1}\right)+\mu_{\phi L} \bar{S}_{2}^{\prime}\left(L-x_{i+1}\right)\right\}+ \\
C_{1} C_{n} \bar{S}_{11}^{\prime \prime}\left(x_{1}\right) \bar{S}_{2}^{\prime \prime}\left(x_{n}-x_{1}\right) \\
\left\{\bar{S}_{2}^{\prime \prime}\left(L-x_{n}\right)+\mu_{\phi L} \bar{S}_{2}^{\prime}\left(L-x_{n}\right)\right\}+ \\
C_{1} \bar{S}_{11}^{\prime \prime}\left(x_{1}\right)\left\{\bar{S}_{2}^{\prime \prime}\left(L-x_{n}\right)+\mu_{\phi L} \bar{S}_{2}^{\prime}\left(L-x_{n}\right)\right\} \\
\prod_{j=2}^{n} C_{j} \bar{S}_{2}^{\prime \prime}\left(x_{j}-x_{j-1}\right) \\
\bar{S}_{22}^{\prime \prime}(L)+\mu_{\phi L} \bar{S}_{22}^{\prime}(L)+\sum_{i=1}^{n} C_{i} \bar{S}_{22}^{\prime \prime}\left(x_{i}\right) \\
\left\{\bar{S}_{2}^{\prime \prime}\left(L-x_{i}\right)+\mu_{\phi L} \bar{S}_{2}^{\prime}\left(L-x_{i}\right)\right\}+ \\
\sum_{j=2}^{n-1} C_{i} C_{i+1} \bar{S}_{22}^{\prime \prime}\left(x_{i}\right) \bar{S}_{2}^{\prime \prime}\left(x_{i+1}-x_{i}\right) \\
\left\{\bar{S}_{2}^{\prime \prime}\left(L-x_{i+1}\right)+\mu_{\phi L} \bar{S}_{2}^{\prime}\left(L-x_{i+1}\right)\right\} \\
C_{1} \bar{S}_{22}^{\prime \prime}\left(x_{1}\right)\left\{\bar{S}_{2}^{\prime \prime}\left(L-x_{n}\right)+\mu_{\phi L} \bar{S}_{2}^{\prime}\left(L-x_{n}\right)\right\} \\
C_{1} C_{n} \bar{S}_{22}^{\prime \prime}\left(x_{1}\right) \bar{S}_{2}^{\prime \prime}\left(x_{n}-x_{1}\right) \\
\left.\bar{S}_{2}^{\prime \prime}\left(L-x_{n}\right)+\mu_{\phi L} \bar{S}_{2}^{\prime}\left(L-x_{n}\right)\right\}+
\end{array}\right] \phi(0)
$$

and

$$
\left[\begin{array}{c}
\bar{S}_{11}^{\prime \prime \prime}(L)-K_{m L} \bar{S}_{11}(L)+\sum_{i=1}^{n} C_{i} \bar{S}_{11}^{\prime \prime}\left(x_{i}\right) \\
\left\{\bar{S}_{2}^{\prime \prime \prime}\left(L-x_{i}\right)-K_{m L} \bar{S}_{2}\left(L-x_{i}\right)\right\}+ \\
\sum_{i=1}^{n-1} C_{i} C_{i+1} \bar{S}_{11}^{\prime \prime}\left(x_{i}\right) \bar{S}_{2}^{\prime \prime}\left(x_{i+1}-x_{i}\right) \\
\left\{\bar{S}_{2}^{\prime \prime}\left(L-x_{i+1}\right)-K_{m L} \bar{S}_{2}\left(L-x_{i+1}\right)\right\}+ \\
C_{1} C_{n} \bar{S}_{11}^{\prime \prime}\left(x_{1}\right) \bar{S}_{2}^{\prime \prime \prime}\left(x_{n}-x_{1}\right) \\
\left\{\bar{S}_{2}^{\prime \prime}\left(L-x_{n}\right)-K_{m L} \bar{S}_{2}\left(L-x_{n}\right)\right\}+ \\
C_{1} \bar{S}_{11}^{\prime \prime}\left(x_{1}\right)\left\{\bar{S}_{2}^{\prime \prime}\left(L-x_{n}\right)-K_{m L} \bar{S}_{2}\left(L-x_{n}\right)\right\} \\
\prod_{j=2}^{n} C_{j} \bar{S}_{2}^{\prime \prime}\left(x_{j}-x_{j-1}\right) \\
\bar{S}_{22}^{\prime \prime \prime}(L)-K_{m L} \bar{S}_{22}(L)+ \\
\sum_{i=1}^{n} C_{i} \bar{S}_{22}^{\prime \prime}\left(x_{i}\right)\left\{\bar{S}_{2}^{\prime \prime \prime}\left(L-x_{i}\right)-K_{m L} \bar{S}_{2}\left(L-x_{i}\right)\right\} \\
\sum_{i=1}^{n-1} C_{i} C_{i+1} \bar{S}_{22}^{\prime \prime}\left(x_{i}\right) \bar{S}_{2}^{\prime \prime}\left(x_{i+1}-x_{i}\right) \\
\left\{\bar{S}_{2}^{\prime \prime \prime}\left(L-x_{i+1}\right)-K_{m L} \bar{S}_{2}\left(L-x_{i+1}\right)\right\}+ \\
C_{1} C_{n} \bar{S}_{22}^{\prime \prime}\left(x_{1}\right) \bar{S}_{2}^{\prime \prime}\left(x_{n}-x_{1}\right) \\
\left\{\bar{S}_{2}^{\prime \prime}\left(L-x_{n}\right)-K_{m L} \bar{S}_{2}\left(L-x_{n}\right)\right\}+ \\
\bar{S}_{1} \bar{S}_{22}^{\prime \prime}\left(x_{1}\right)\left\{\bar{S}_{2}^{\prime \prime \prime}\left(L-x_{n}\right)-K_{m L} \bar{S}_{2}\left(L-x_{n}\right)\right\} \\
n \\
j=2
\end{array}\right] \phi(0)
$$$$
=0 .
$$

The frequency equation is obtained by setting the second-order determinant formed from the coefficients of $X(0)$ and $\phi(0)$ in Eqns (19) and (20) to zero. The eigen frequency $\omega_{j}(j=1,2,3, \ldots)$ is then calculated from the frequency equation. Substituting the calculated value of $\omega_{j}$ back into Eqns (19) and (20) and setting either $X(0)$ or $\phi(0)$ to 1 or any other value, the $j^{\text {th }}$ mode shape can be obtained. The first derivative of the mode shape yields the mode shape rotation, from which the mode shape rotation deviation curve can readily be determined.

\section{Generation of training and test data for ANN and its architecture}

The characteristic equation is determined from Eqns (19) and (20), which is then analytically solved to obtain natural frequencies of beam structures. These equations are highly non-linear and take too much CPU time on regular computers. Therefore, the analyses have been run on Intel Xeon E5645 machine with two CPUs of $2.4 \mathrm{GHz}$ with 
12 cores, 24 threads and $48 \mathrm{~GB}$ ram. The values of the rectilinear and rotational spring stiffnesses in these equations can be assigned in a way that any beam configuration of desire is acquired. For example, a free end is attained by giving zero stiffness to spring elements. Full fixity can be obtained by using a large value of spring constants. One should be careful, however, about assigning a large value since this value can cause numerical instability during the solution of the characteristic equation. The rule of thumb for choosing spring stiffness to get a full fixity is that the value should be approximately a thousand times greater than the stiffness of the beam element (Hoit 1994) to which the spring is connected in the direction under consideration. It is noted that this approach is approximate. The choice of spring stiffnesses is checked in this study by comparing the natural frequencies of spring-spring supported beams with those of classically supported beams. Consequently, the amount to be used for full fixity is determined. A value of zero is utilized for free DOF and a normalized value of 1 for full fixity. This results in 16 beam types in total. Intermediate quantities of spring constants can also be used but in order not to make the ANN architecture too complex, this study considers these two limitingvalues. Some of the resulting example beam structures are presented in Figure 2.

As stated previously, the beam material and geometrical characteristics have not been used in the literature of crack identification through ANN-based methods. Only one study, which is actually not damage identification but a study of determination of vibrational frequencies of intact beams via ANN (Civalek 2004), considered these variables. The study herein is hence the first in that respect. The minimum and maximum values of beam parameters utilized are given in Table 1.

It is reported in the current literature that the ANNs can learn about the response of undamaged beams. This study, therefore, examined first the feasibility of ANNs

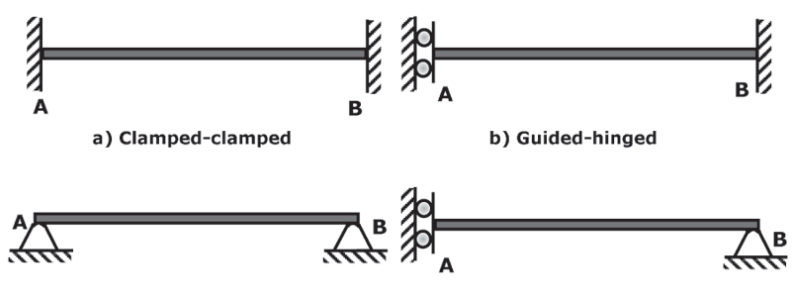

$\begin{array}{ll}\text { c) Hinged-hinged } & \text { d) Guided-hinged }\end{array}$

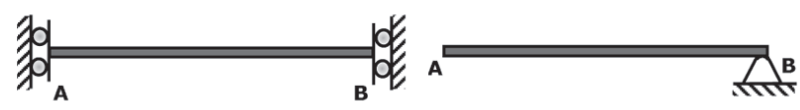

$\begin{array}{ll}\text { e) Guided-guided } & \text { f) Free-hinged }\end{array}$

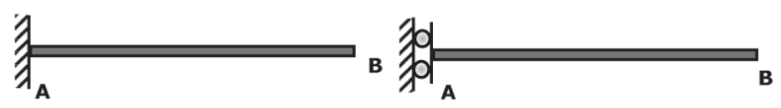

g) Clamped-free

h) Guided-free

Fig. 2. Examples of the beams resulting from the extreme conditions of spring stiffnesses
Table 1. Ranges of beam material and geometrical properties used in training the neural network

\begin{tabular}{lccc}
\hline Parameter & $\begin{array}{c}\text { Minimum } \\
\text { value }\end{array}$ & $\begin{array}{c}\text { Maximum } \\
\text { value }\end{array}$ & $\begin{array}{c}\text { Number of } \\
\text { parameter } \\
\text { within range }\end{array}$ \\
\hline $\begin{array}{l}\text { Height of beam } \\
h(\mathrm{~m})\end{array}$ & 0.05 & 0.45 & 9 \\
\hline $\begin{array}{l}\text { Mass density } \\
\rho\left(\mathrm{kg} / \mathrm{m}^{3}\right)\end{array}$ & 500 & 8000 & 18 \\
\hline $\begin{array}{l}\text { Length of beam } \\
L(\mathrm{~m})\end{array}$ & 0.5 & 4.5 & 9 \\
\hline $\begin{array}{l}\text { Modulus of } \\
\text { elasticity } \\
E(\mathrm{GPa})\end{array}$ & 29 & 220 & 11 \\
\hline
\end{tabular}

in the forward problems, i.e. the determination of natural frequencies of intact beams given the support conditions, material and geometrical properties. Accordingly, a total of 64152 different beam configurations are obtained and used as input to the neural networks in the forward problem.

The above beam configurations are introduced with local damage for the inverse problem. A number of simulations are performed for various crack depths at different locations. The crack location $x_{1}$ is varied from $0.05 \mathrm{~L}$ to $1.0 L$ in steps of 0.05 and crack depth $a_{1}$ is varied from $0.05 h$ to $0.5 h$ in steps of 0.05 . This results in 200 different damage scenarios. Due to the symmetry between the natural frequencies and crack parameters for some beam structures, an additional parameter is needed in uniquely identifying the crack. Modal shapes and their derivatives have been frequently employed in the past. This study uses mode shape rotation deviation curves for that purport. These curves are computed from a simple transformation of the mode shape rotation data. Let $\phi_{i}$ be the $i^{\text {th }}$ datum of mode shape rotation along the beam length. Then:

$$
\overline{\phi_{i}}=\phi_{i+1}-\phi_{i}
$$

yields the desired deviation data. Combination of these deviation data with natural frequencies, the beam types, beam material and geometrical properties produce the data input to train the ANN of the inverse identification problem.

\section{Neuralnetwork models}

\subsection{Multi-layer perceptron (MLP)}

The multi-layer perceptron (MLP) is based on the present understanding of the biological nervous system. It is a massive parallel system composed of many processing elements connected by links of variable weights. Among the many MLP paradigms, the back propagation network is by far the most popular (Haykin 1998). The network consists of layers of parallel processing units or neurons. Each layer is connected to the proceeding layer by interconnection strengths or weights, $W$. Figure 3 illustrates a three-layered MLP network consisting of layers $i, j$ and $k$ with the interconnection weights $W_{i j}$ and $W_{j k}$ between 
the layers of the neurons. The initial assigned weights are progressively corrected during the training process. In this process, the outputs predicted by MLP are compared with known outputs, and errors are back propagated (from right to left in Fig. 3) to determine the appropriate weight adjustments necessary to minimize errors. In this study, the Levenberg-Marquardt algorithm (Marquardt 1963) is used for adjusting the MLP weights (Hagan et al. 1994; Kisi 2004; Kocabas et al. 2008, 2010a; Kisi, Ay 2011). Detailed information about MLP can be found in Haykin (1998).

\subsection{Radial basis neural network (RBNN)}

RBNN was first introduced into the ANN literature by Broomhead and Lowe (1988) and Poggio and Girosi (1990). The RBNN has two layers whose output nodes form a linear combination of the basis functions. RBNN is also known as a localized receptive field network because of the fact that the basis functions in the hidden layer produce a significant nonzero response to input stimulus only when the input falls within a small localized region of the input space (Lee, Chang 2003). The relation between inputs and outputs is demonstrated in Figure 4. The RBNN has connection weights between the hidden layer and the output layer only. These weight values can be obtained by the linear least-squares method, which gives an important advantage for convergence. The Gaussian activation function is widely used as a radial basis function. The RBNN can be considered as a special case of MLR. The RBNN method does not perform parameter learning as in MLP. It performs linear adjustment of the weights for the radial bases. This characteristic gives RBNN the advantage of a very fast converging time without local minima (Kocabas et al. 2010b; Kisi, Ay 2011) because its error function is always a convex. In this study, different numbers of hidden layer neurons are examined for the RBNN models with a simple trialand-error method. Detailed information about the RBNN method can be obtained from Haykin (1998).

\section{Applications of neural network models}

This section presents the design and predictions of neural network models in forward and inverse problems. Due to a very large number of input parameters, the ability of

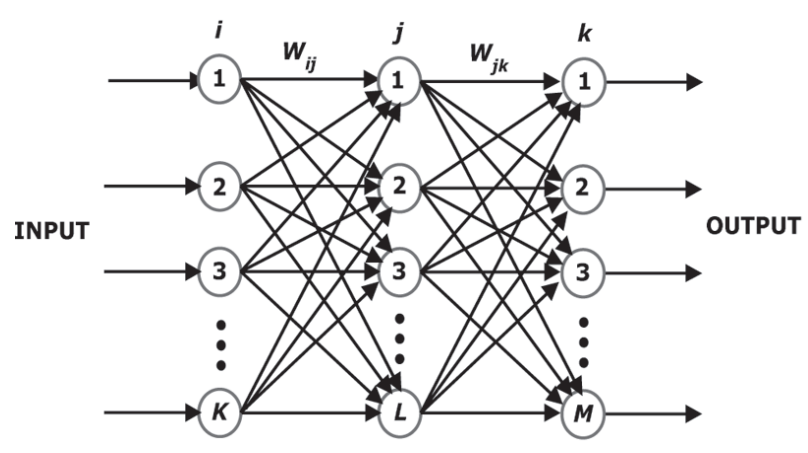

Fig. 3. Schematic diagram of MLP architecture

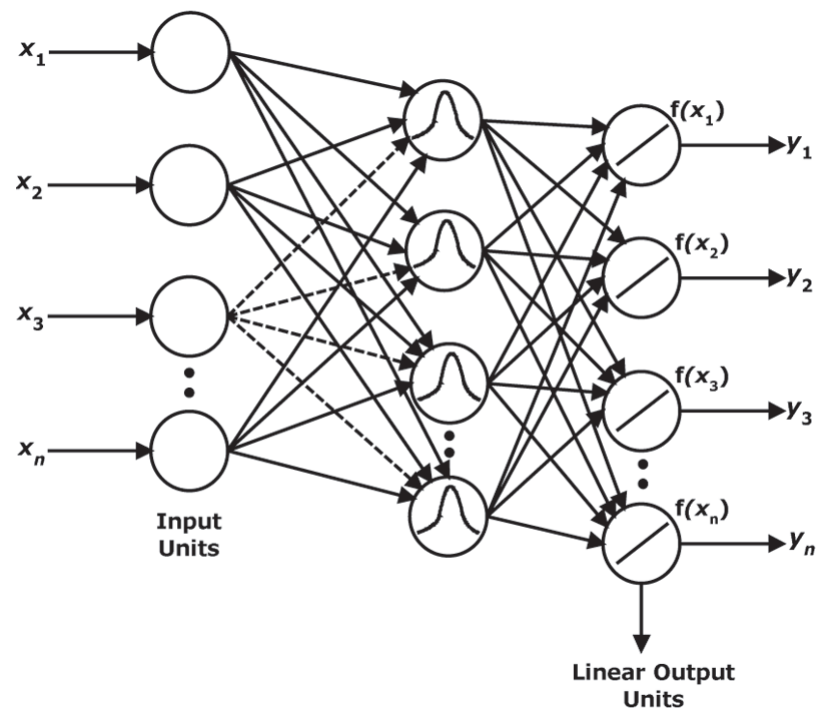

Fig. 4. Schematic diagram of RBNN architecture

the ANNs to estimate the vibrational frequencies of intact beams given the material properties, beam geometrical properties and support conditions is first examined. The inverse problem of identifying the crack location and depth is later studied. For these purposes, two different codes in MATLAB including Neural Network Toolbox are written for the MLP and RBNN simulations. Each simulation considers a variety of models to determine the most effective neural networks. In order to evaluate the efficiency of each model, mean absolute relative error (MARE), root mean square error (RMSE) and determination coefficient $\left(\mathrm{R}^{2}\right)$ statistics are used. The $\mathrm{R}^{2}$ measures the degree to which two variables are linearly related. MARE and RMSE provide different types of information about the predictive capabilities of the model. The RMSE measures the goodness-of-fit relevant to high values whereas the MARE yields a more balanced perspective of the goodness-of-fit at moderate values (Karunanithi et al. 1994). The optimal model should have the minimal RMSE and MARE and an $\mathrm{R}^{2}$ close to 1 . The RMSE, MARE, and $\mathrm{R}^{2}$ statistics are given by:

$$
\begin{gathered}
\text { MARE }=\frac{1}{N} \sum_{i=1}^{N}\left|\frac{Y_{O, i}-Y_{M, i}}{Y_{O, i}}\right| 100 \\
\operatorname{RMSE}=\frac{1}{N} \sqrt{\sum_{i=1}^{N}\left(Y_{O, i}-Y_{M, i}\right)^{2}} ; \\
\mathrm{R}^{2}=\left(\frac{\sum_{i=1}^{N}\left(Y_{O, i}-\bar{Y}_{O}\right) \sum_{i=1}^{N}\left(Y_{M, i}-\bar{Y}_{M}\right)}{\sqrt{\sum_{i=1}^{N}\left(Y_{O, i}-\bar{Y}_{O}\right) \sum_{i=1}^{N}\left(Y_{M, i}-\bar{Y}_{M}\right)^{2}}}\right)^{2},
\end{gathered}
$$


where: $N$ is the number of observations; $Y_{o, i}$ is the $i^{\text {th }}$ observed datum; $Y_{M, i}$ is the $i^{\text {th }}$ corresponding simulated datum; $\bar{Y}_{O}$ and $\bar{Y}_{M}$ are mean of the observed and simulated values. Before applying the MLP and RBNN models to the data, the training input and output values were normalized using the equation:

$$
a \frac{x_{i}-x_{\min }}{x_{\max }-x_{\min }}+b
$$

where $x_{\min }$ and $x_{\max }$ are the minimum and maximum of the data set. In this study, $a$ and $b$ are taken as 0.6 and 0.2 following the suggestion of Cigizoglu (2003). According to the Cigizoglu (2003), normalizing data in the range $[0.2,0.8]$ increases the flexibility of ANN models.

In the first part of the study, the first four natural frequencies of beam elements are predicted based on beam material, geometrical and boundary conditions. Accordingly, a total of seven inputs and four outputs are used in training the neural network models. Due to a high number of input data, the simulations are run on previously mentioned Intel Xeon E565 machine. Several hidden node numbers are tried for the MLP and RBNN models. For each hidden node number, various numbers of iterations are tried for the MLP models. The iteration numbers tried for each MLP model are 5000, 10000, 20000, 30000, 40000 and 50000. For the RBNN models the optimal spread constant value for each RBNN model is obtained. The spread values tried for each RBNN model are $0.1,0.2 \ldots 2.0$. Optimum parameters of the MLP and RBNN models are determined by minimizing the objective function (MARE between calculated and observed frequency values) in test period. The test results of the optimal MLP models in frequency estimation are given in Table 2 . The first three columns of Table 2 indicate that the optimum iteration number increases parallel to the hidden node numbers. It is clear from the table that the MLP5 model which has 50 hidden nodes with 20000 iterations performs better than the other models. MLP7 model has better accuracy than the MLP5 in estimating the first, the third and the fourth frequency values. However, it seems to be insufficient in estimating the frequency of the second mode.

Table 3 displays the test results of the optimal RBNN models in frequency estimation. It can be clearly seen from the table that the RBNN7 model comprising 200 hidden nodes with 0.8 spread value performs better than the other models. Comparison of Tables 2 and 3 reveals that the optimal RBNN model (RBNN7) performs better than the optimal MLP5 model in estimating frequency values. The estimates of the MLP and RBNN models in test period are shown in Figure 5. The fit line equations and $\mathrm{R}^{2}$ values of each model are also provided in this figure. It can be seen from the figure that the RBNN model performs better than the MLP model. As seen from the fit line equations (assume that the equation is $\left.y=a_{0} x+a_{1}\right)$ in the scatterplots that the $a_{\mathrm{o}}$ and $a_{1}$ coefficients for the RBNN model are respectively closer to the 1 and 0 with a higher $\mathrm{R}^{2}$ value than those of the MLP model.

In the second part of the study, the inverse problem is dealt with. Crack parameters, i.e. crack location and crack severity, are predicted using the beam properties and

Table 2. The test results of the MLP models in natural frequency estimation

\begin{tabular}{|c|c|c|c|c|c|c|c|c|}
\hline \multirow{2}{*}{$\begin{array}{c}\text { Hidden } \\
\text { node number }\end{array}$} & \multirow{2}{*}{ Model } & \multirow{2}{*}{ Epochs } & \multicolumn{3}{|c|}{ Frequency of mode 1} & \multicolumn{3}{|c|}{ Frequency of mode 2} \\
\hline & & & MARE & RMSE & $\mathrm{R}^{2}$ & MARE & RMSE & $\mathrm{R}^{2}$ \\
\hline 10 & MLP1 & 5000 & 89.5 & 2113 & 0.001 & 8.39 & 343.3 & 0.996 \\
\hline 20 & MLP2 & 5000 & 5.49 & 65.24 & 0.999 & 84.8 & 6926 & 0.022 \\
\hline 30 & MLP3 & 10000 & 3.43 & 60.59 & 0.999 & 3.07 & 123.8 & 0.999 \\
\hline 40 & MLP4 & 20000 & 6.17 & 75.67 & 0.998 & 84.8 & 6926 & 0.003 \\
\hline 50 & MLP5 & 20000 & 7.48 & 82.43 & 0.998 & 5.58 & 216.4 & 0.999 \\
\hline 100 & MLP6 & 50000 & 4.06 & 32.49 & 0.999 & 84.8 & 6926 & 0.001 \\
\hline 200 & MLP7 & 50000 & 3.94 & 45.58 & 0.999 & 84.8 & 6926 & 0.016 \\
\hline \multirow{2}{*}{$\begin{array}{c}\text { Hidden } \\
\text { node number }\end{array}$} & \multirow{2}{*}{ Model } & \multirow{2}{*}{ Epochs } & \multicolumn{3}{|c|}{ Frequency of mode 3} & \multicolumn{3}{|c|}{ Frequency of mode 4} \\
\hline & & & MARE & RMSE & $\mathrm{R}^{2}$ & MARE & RMSE & $\mathrm{R}^{2}$ \\
\hline 10 & MLP1 & 5000 & 6.98 & 596.0 & 0.998 & 7.35 & 1173 & 0.997 \\
\hline 20 & MLP2 & 5000 & 3.04 & 335.0 & 0.999 & 81.8 & 26220 & 0.009 \\
\hline 30 & MLP3 & 10000 & 82.9 & 14957 & 0.001 & 3.19 & 611.2 & 0.999 \\
\hline 40 & MLP4 & 20000 & 82.9 & 14957 & 0.004 & 3.75 & 795.3 & 0.999 \\
\hline 50 & MLP5 & 20000 & 5.17 & 469.4 & 0.998 & 4.91 & 878.6 & 0.998 \\
\hline 100 & MLP6 & 50000 & 2.14 & 195.2 & 0.999 & 81.8 & 26220 & 0.001 \\
\hline 200 & MLP7 & 50000 & 2.02 & 177.4 & 0.9997 & 2.12 & 361.7 & 0.9997 \\
\hline
\end{tabular}


Table 3. The test results of the RBNN models in natural frequency estimation

\begin{tabular}{|c|c|c|c|c|c|c|c|c|}
\hline \multirow{2}{*}{$\begin{array}{c}\text { Hidden } \\
\text { node number }\end{array}$} & \multirow{2}{*}{ Model } & \multirow{2}{*}{$\begin{array}{l}\text { Spread } \\
\text { value }\end{array}$} & \multicolumn{3}{|c|}{ Frequency of mode 1} & \multicolumn{3}{|c|}{ Frequency of mode 2} \\
\hline & & & MARE & RMSE & $\mathrm{R}^{2}$ & MARE & RMSE & $\mathrm{R}^{2}$ \\
\hline 10 & RBN1 & 0.8 & 31.1 & 660.7 & 0.867 & 33.9 & 2281 & 0.840 \\
\hline 20 & RBN2 & 0.9 & 39.3 & 541.6 & 0.910 & 28.5 & 1668 & 0.913 \\
\hline 30 & RBN3 & 1.1 & 37.5 & 287.0 & 0.978 & 14.9 & 840.0 & 0.978 \\
\hline 40 & RBN4 & 0.9 & 30.4 & 229.4 & 0.984 & 13.0 & 731.6 & 0.983 \\
\hline 50 & RBN5 & 1.0 & 22.6 & 204.5 & 0.987 & 12.2 & 1457 & 0.987 \\
\hline 100 & RBN6 & 0.8 & 8.72 & 94.35 & 0.997 & 6.82 & 335.0 & 0.996 \\
\hline 200 & RBN7 & 0.8 & 3.48 & 33.89 & 0.9997 & 2.65 & 121.5 & 0.9995 \\
\hline \multirow{2}{*}{$\begin{array}{c}\text { Hidden } \\
\text { node number }\end{array}$} & \multirow{2}{*}{ Model } & \multirow{2}{*}{$\begin{array}{l}\text { Spread } \\
\text { value }\end{array}$} & \multicolumn{3}{|c|}{ Frequency of mode 3} & \multicolumn{3}{|c|}{ Frequency of mode 4} \\
\hline & & & MARE & RMSE & $\mathrm{R}^{2}$ & MARE & RMSE & $\mathrm{R}^{2}$ \\
\hline 10 & RBN1 & 0.8 & 36.0 & 5228 & 0.813 & 36.8 & 9506 & 0.796 \\
\hline 20 & RBN2 & 0.9 & 29.1 & 3594 & 0.911 & 30.0 & 6387 & 0.907 \\
\hline 30 & RBN3 & 1.1 & 14.1 & 1852 & 0.976 & 15.6 & 3405 & 0.974 \\
\hline 40 & RBN4 & 0.9 & 14.1 & 1758 & 0.979 & 16.4 & 3331 & 0.975 \\
\hline 50 & RBN5 & 1.0 & 12.4 & 1457 & 0.985 & 13.5 & 2706 & 0.983 \\
\hline 100 & RBN6 & 0.8 & 8.29 & 864.4 & 0.995 & 9.00 & 1678 & 0.994 \\
\hline 200 & RBN7 & 0.8 & 3.09 & 304.9 & 0.9994 & 3.32 & 584.2 & 0.9992 \\
\hline
\end{tabular}

dynamic-based analysis data. Hence, a total of 15 input parameters are utilized to determine two output quantities. Here also several hidden node numbers are tried for the MLP and RBNN models. The iteration numbers tried for each MLP model are 1000, 5000 and 10000. Epochs values greater than 10000 produce out-of-memory errors in the simulations, hence, could not be used in the identification problem. The same spread values $(0.1,0.2 \ldots 2.0)$ used in the forward problem are alsoattempted for each RBNN model of crack identification case. The optimal MLP models'test results in crack estimation are given in Table 4 . The table evidently shows that the MLP2 model that has 100 hidden nodes with 10000 iterations performs better than the other MLP models. The test results of the optimal RBNN models in crack estimation are illustrated in Table 5. It is observed from the table that the RBNN9 model comprising 2000 hidden nodes with 0.7 spread value performs better than the other RBNN models. Comparison of Tables 4 and 5 indicates that the optimal RBNN model (RBNN9) performs much better than the optimal MLP2 model in estimating crack. Figure 6 shows the estimates of crack location of RBNN and MLP models in test period in the form of time series data. It can be seen that the neural network models closely follow the actual data with no over-fitting. Figure 7 compares the crack severity predictions of optimal MLP and RBNN models in test period. It is seen from the figure that the MLP estimates are more scattered than those of the RBNN model. Since the RBNN has a better interpolating capability in the multidimensional space, it can approximate the crack parameters better than the MLP (Haykin 1998).

\subsection{Effect of noise on the performance of neural network models}

In order to simulate the uncertainties of real-life problems and to get a better generalization during the training of ANNs in the crack identification case, artificial random noise is added to the normalized vibrational frequencies and mode shape rotation deviation values. Noise with zero mean and unit standard deviation is added to the dynamic data as follows:

$$
x_{\text {noise }}=x(1+S F \cdot y),
$$

where: $x_{\text {noise }}$ is the data with noise; $x$ is the original data without noise; $S F$ is the scale factor (or noise level); and $y$ is the randomly generated noise vector. Three different noise levels, i.e. 1, 2 and $3 \%$ are considered. The same MLP and RBNN models that have the lowest error estimates in noise-free cases are again used herein for training and validation. The results for additional artificial noise cases are given in Table 6 for performance test. It is observed that as the noise level increases, the predictions of ANN models get worse. When the ANN estimates are compared to those of the previous studies that considered noise, the errors here are a little bit higher. However, keeping in mind that those studies dealt with only one case of beam and a single set of beam material and geometrical properties and this study covered a great variety of beam types, these relative errors should be acceptable. Another observation is made from the table that when the gradually increased noise combination applied to the vibrational frequencies and mode shape rotation deviation quantities are used in order to predict 

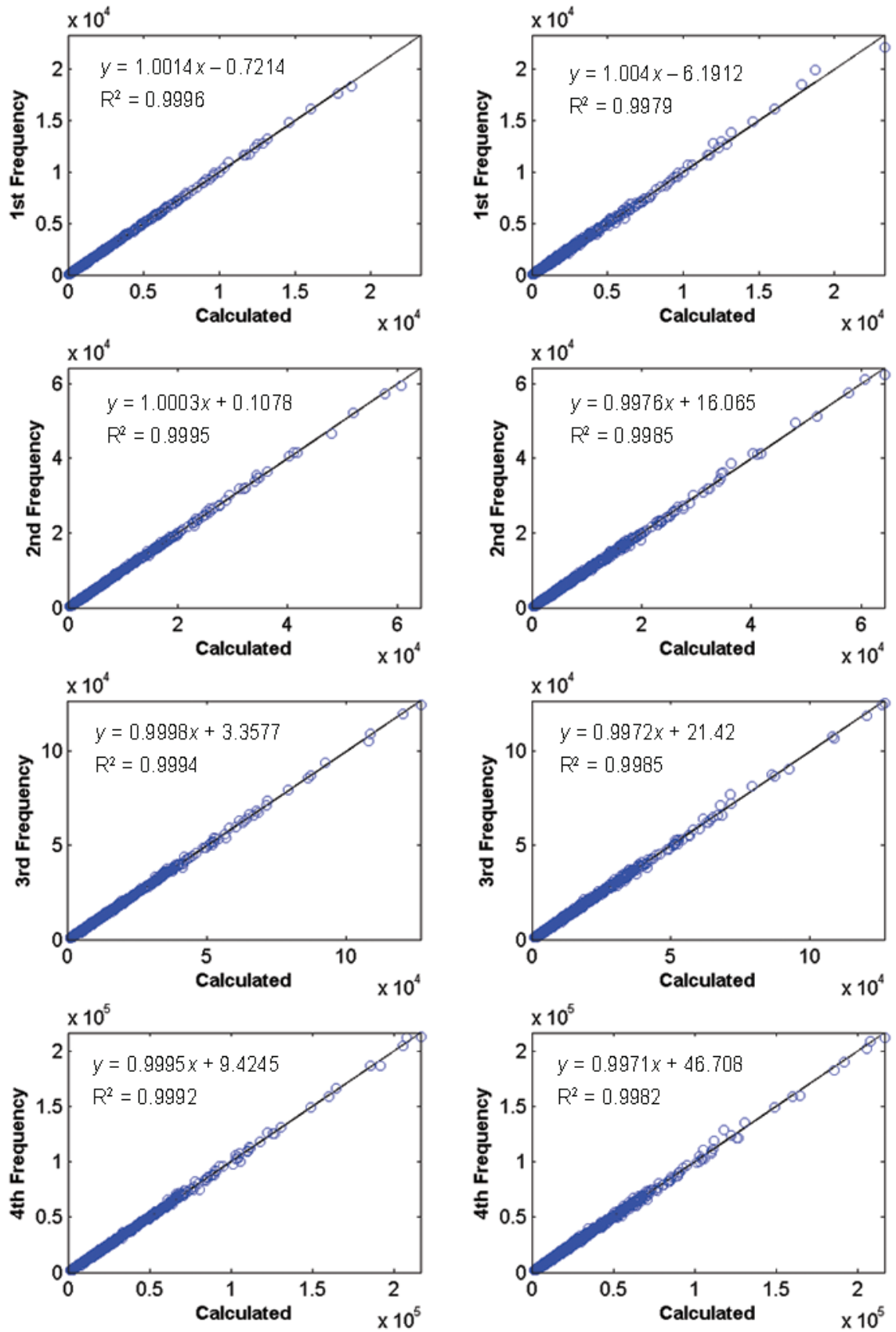

Fig. 5. Calculated and modelled frequencies by RBNN (left) and MLP (right) models in test phase

Table 4. The test results of the MLP models in estimation of crack parameters

\begin{tabular}{ccccccccc}
\hline \multirow{2}{*}{$\begin{array}{c}\text { Hidden } \\
\text { node number }\end{array}$} & Model & Epochs & \multicolumn{3}{c}{ Crack Location } & \multicolumn{3}{c}{ Crack Depth } \\
\cline { 4 - 9 } & & MARE & RMSE & $\mathrm{R}^{2}$ & MARE & RMSE & $\mathrm{R}^{2}$ \\
\hline 50 & MLP1 & 10000 & 48.1 & 0.294 & 0.901 & 44.4 & 0.291 & 0.903 \\
\hline $\mathbf{1 0 0}$ & MLP2 & $\mathbf{1 0 0 0 0}$ & $\mathbf{4 8 . 6}$ & $\mathbf{0 . 2 8 1}$ & $\mathbf{0 . 9 0 9}$ & $\mathbf{4 5 . 9}$ & $\mathbf{0 . 2 9 2}$ & $\mathbf{0 . 9 0 2}$ \\
\hline 500 & MLP3 & 10000 & 53.4 & 0.938 & 0.501 & 53.6 & 0.926 & 0.509 \\
\hline 1000 & MLP4 & 10000 & 53.7 & 0.938 & 0.497 & 53.8 & 0.927 & 0.499 \\
\hline
\end{tabular}


Table 5. The test results of the RBNN models in estimation of crack parameters

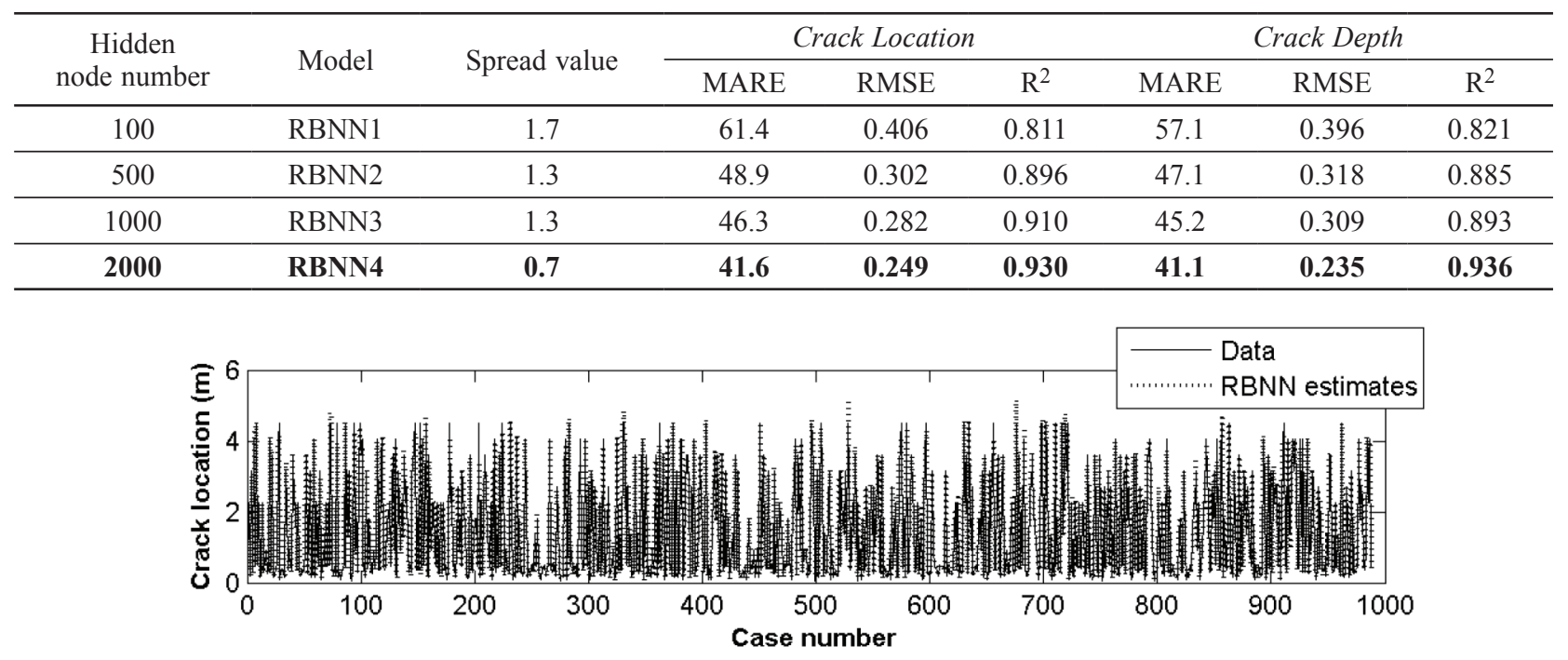
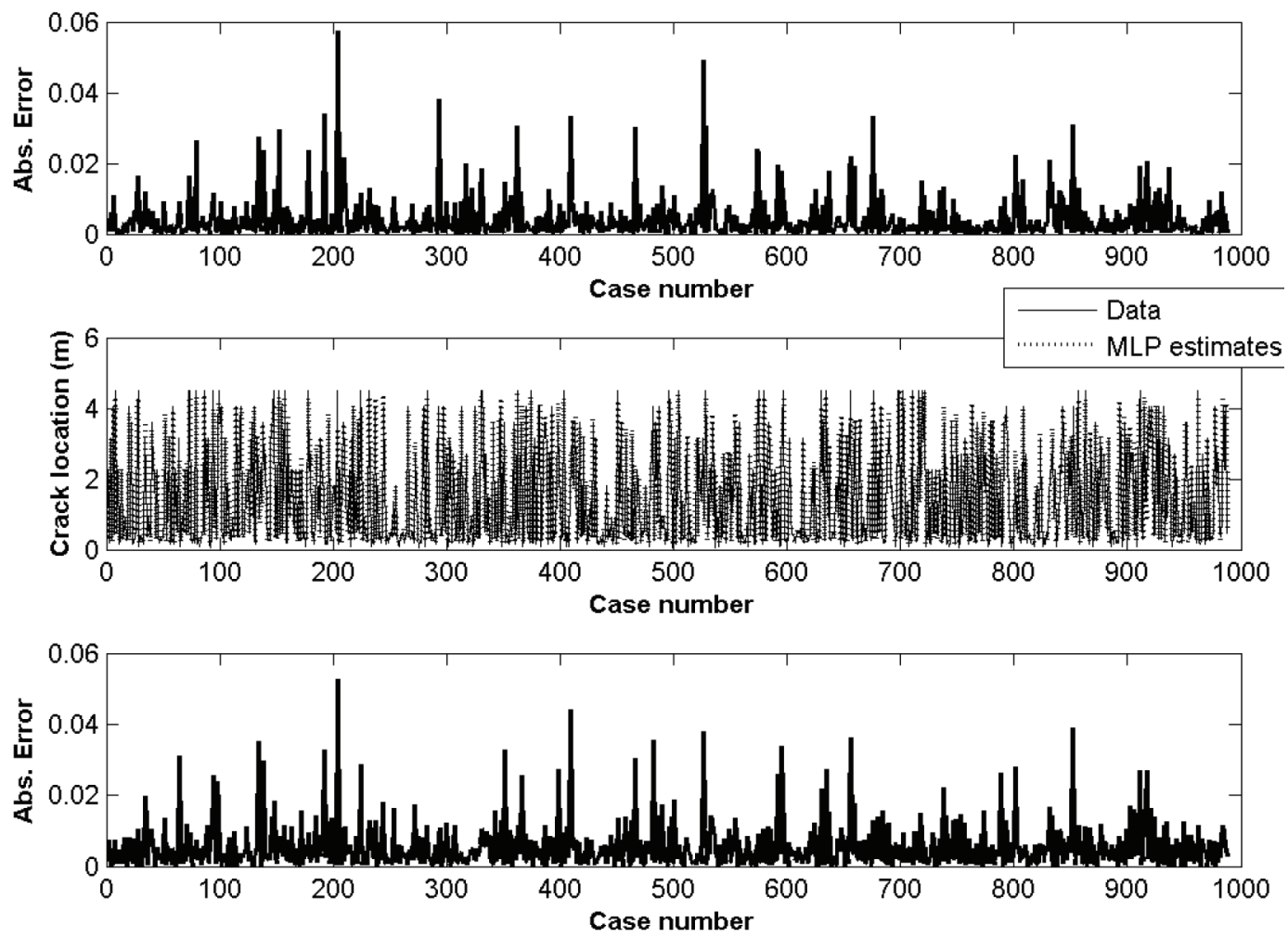

Fig. 6. The RBNN and MLP estimates of crack location and absolute errors in model predictions
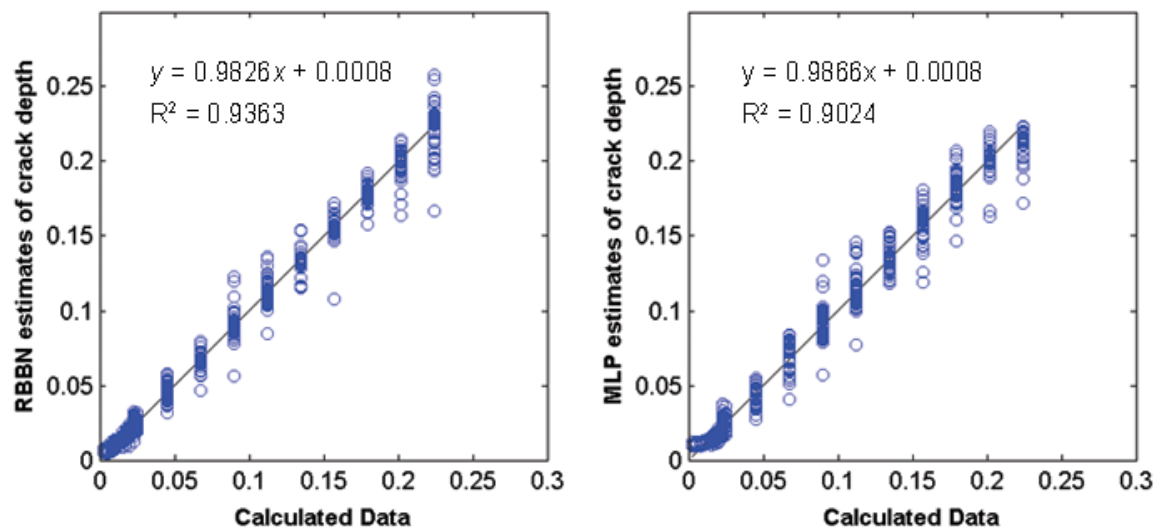

Fig. 7. Calculated and modelled crack severity by RBNN and MLP models in test phase 
Table 6. Predictions of ANN models in the presence of noise

\begin{tabular}{|c|c|c|c|c|c|c|c|c|c|c|}
\hline \multirow[b]{2}{*}{$\begin{array}{l}\text { Noise } \\
\text { level }\end{array}$} & \multirow[b]{2}{*}{$\begin{array}{c}\text { Crack } \\
\text { location } \\
\left(x_{1} / L\right)\end{array}$} & \multirow[b]{2}{*}{$\begin{array}{c}\text { Crack } \\
\text { severity } \\
(a / h)\end{array}$} & \multicolumn{4}{|c|}{ MLP prediction } & \multicolumn{4}{|c|}{ RBNN prediction } \\
\hline & & & Location & $\begin{array}{l}\text { Rel.error } \\
(\%)\end{array}$ & Severity & $\begin{array}{l}\text { Rel. error } \\
\quad(\%)\end{array}$ & Location & $\begin{array}{l}\text { Rel. } \\
\text { error } \\
(\%)\end{array}$ & Severity & $\begin{array}{l}\text { Rel. } \\
\text { error } \\
(\%)\end{array}$ \\
\hline $1 \%$ & 0.65 & 0.05 & 0.6318 & 2.8 & 0.0494 & 1.2 & 0.5862 & 9.8 & 0.0498 & 0.4 \\
\hline $2 \%$ & 0.65 & 0.05 & 0.6142 & 5.5 & 0.0486 & 2.8 & 0.5614 & 13.6 & 0.0489 & 2.2 \\
\hline $3 \%$ & 0.65 & 0.05 & 0.5298 & 18.5 & 0.0470 & 5.9 & 0.5272 & 18.9 & 0.0468 & 6.3 \\
\hline
\end{tabular}

the severity and location of cracks, better estimates are obtained in severity predictions compared to the location predictions at each level of noise. This is attributed to non-uniqueness of the relation between the crack parameters and vibrational data.

\section{Conclusions}

Two neural network approaches are examined to estimate the vibrational frequencies of pristine beams in the forward problem and crack parameters (location and depth) in the inverse problem. Considering various beam material properties, beam geometrical characteristics and beam boundary conditions, a large number of input data sets are produced based on the theoretical analysis of beam elements. The theory to obtain the dynamic data (natural frequencies and mode shape rotation deviation curves) is provided. In the forward problem, four support conditions and three beam properties (e.g. modulus of elasticity, mass density and length of beam) are used as input to the neural network models to predict the first four natural frequencies of beam structures. In addition to these parameters, the first four natural frequencies and mode shape rotation deviation data (15 in total) are employed as input to the network models in the inverse problem to estimate the location and severity of cracks present in beam structures. Training the data sets are carried out using a multi-layer perceptron and a radial basis function networks. Although a great number of input data, no data reduction techniques have been employed. In the forward problem, the MLP model that has 50 hidden nodes with 20000 iterations and the RBNN model that has 200 hidden nodes with a spread value of 0.8 have performed better than the other corresponding models. Comparison of the MLP and RBNN error estimates yields that the optimal RBNN model accomplish better than the optimal MLP model. The same conclusion is also drawn in the inverse problem of predicting the crack parameters. In this case, the MLP model with 50 hidden nodes and 10000 iterations, and the RBNN model with 2000 hidden nodes and 0.7 spread valueperformed better. That the RBNN model predicts the crack parameters better the optimal MLP model is attributed to the fact that the RBNN has a better interpolating capability in multidimensional space. Though the optimal RBNN yields better estimations in both cases, it takes slightly longer time in training the input data. The robustness of the optimal MLP and RBNN models in the presence of noise is also investigated. For this purpose, a random noise with zero mean and unit standard deviation is added to the vibration data. Comparison of the current estimates with the literature values shows that the errors of this study are a bit higher. However, this increase is within acceptable range and is due to the non-uniqueness of the relationship between the input and output data sets and the very large number of beam cases considered. The relative errors of optimal MLP model are somewhat less than those of the optimal RBNN model.

As a conclusion, the trained RBNN model can be used in health monitoring of beam-like structures as a non-destructive crack identification procedure. Although it is very hard to accumulate such large number of input data, the developed network should be tested using the real measurement cases.

\section{References}

Abdel Wahab, M. M.; De Roeck, G. 1999. Damage detection in bridges using modal curvatures: application to a real damage scenario, Journal of Sound and Vibration 226(2): 217-235. http://dx.doi.org/10.1006/jsvi.1999.2295

Abdo, M. A. B.; Hori, M. 2002. A numerical study of structural damage detection using changes in the rotation of mode shapes, Journal of Sound and Vibration 251(2): 227-239. http://dx.doi.org/10.1006/jsvi.2001.3989

Bakhary, N.; Hao, H.; Deeks, A. 2007. Damage detection using artificial neural network with consideration of uncertainties, Engineering Structures 29(11): 2806-2815. http://dx.doi.org/10.1016/j.engstruct.2007.01.013

Bakhary, N. 2008. Structural condition monitoring and damage identification with artificial neural network. PhD thesis. Perth, The University of Western Australia, Perth, WA, Australia.

Broomhead, D.; Lowe, D. 1988. Multivariable functional interpolation and adaptive networks, Complex Systems 2(6): 321-355.

Carden, E. P.; Fanning, P. 2004. Vibration based condition monitoring: areview, Structural Health Monitoring 3(4): 355-377. http://dx.doi.org/10.1177/1475921704047500

Cigizoglu, H. K. 2003. Estimation, forecasting and extrapolation of flow data by artificial neural networks, Hydrological Sciences Journal 48(3): 349-361. http://dx.doi.org/10.1623/hysj.48.3.349.45288

Civalek, O. 2004. Flexural and axial vibration analysis of beams with different support conditions using artificial neural networks, Structural Engineering and Mechanics 18(3): 303-314. http://dx.doi.org/10.12989/sem.2004.18.3.303

Das, H. C.; Parhi, D. R. 2009. Application of neural network for fault diagnosis of cracked cantilever beam, in Proc. of World Congress on Nature and Biologically Inspired Computing, NaBIC 2009, 9-11 December 2009, Coimbatore, India, 1303-1308. 
Dimarogonas, A. D. 1996. Vibration of cracked structures: astate of the art review, Engineering Fracture Mechanics 55(5): 831-857. http://dx.doi.org/10.1016/0013-7944(94)00175-8

Doebling, S. W.; Farrar, C. R.; Prime, M. B.; Shevitz, D. W. 1996. Damage identification and health monitoring of structural and mechanical systems from changes in their vibration characteristics: aliterature review. Report No: LA-13070-MS, Los Alamos National Laboratory.

Fan, W.; Qiao, P. 2011. Vibration-based damage identification methods: are view and comparative study, Structural Health Monitoring 10(1): 83-111. http://dx.doi.org/10.1177/1475921710365419

Gawronski, W.; Sawicki, J. T. 2000. Structural damage detection using modal norms, Journal of Sound and Vibration 229(1): 194-198. http://dx.doi.org/10.1006/jsvi.1999.2179

Gudmundson, P. 1982. Eigen frequency changes of structures due to cracks, notches or other geometrical changes, Journal of the Mechanics and Physics of Solids 30(5): 339353. http://dx.doi.org/10.1016/0022-5096(82)90004-7

Hagan, M. T.; Menhaj, M. B. 1994. Training feed forward networks with the Marquardt algorithm, IEEE Transactions on Neural Networks 5(6): 861-867. http://dx.doi.org/10.1109/72.329697

Haykin, S. 1998. Neural networks: a comprehensive foundation. Upper Saddle River: Prentice Hall. 842 p.

Hoit, M. I. 1994. Computer assisted structural analysis and modeling. Upper Saddle River: Prentice Hall. 440 p.

Karunanithi, N.; Grenney, W. J.; Whitley, D.; Bovee, K. 1994. Neural networks for river flow prediction, Journal of Computing in Civil Engineering ASCE 8(2): 201-220. http://dx.doi.org/10.1061/(ASCE)0887-3801(1994)8: 2(201)

Kasper, D. G.; Swanson, D. C.; Reichard, K. M. 2008. Higherfrequency wave number shift and frequency shift in a cracked, vibrating beam, Journal of Sound and Vibration 312(1-2): 1-18. http://dx.doi.org/10.1016/j.jsv.2007.07.092

Kawiecki, G. 2001. Modal damping measurement for damage detection, Smart Materials and Structures 10(3): 466-471. http://dx.doi.org/10.1088/0964-1726/10/3/307

Kisi, O. 2004. Multi-layer perceptrons with LevenbergMarquardt training algorithm for suspended sediment concentration prediction and estimation, Hydrological Sciences Journal 49(6): 1025-1040. http://dx.doi.org/10.1623/hysj.49.6.1025.55720

Kisi, O.; Ay, M. 2011. Modeling dissolved oxygen (DO) concentration using different neural network technique, in Proc. of International Balkans Conference on Challenges of Civil Engineering, BCCCE, 19-21 May 2011, Epoka University, Tirana, Albania, 122-128.

Kocabas, F.; Unal, S.; Unal, B. 2008. A neural network approach for prediction of critical submergence of an intake in still water and open channel flow for permeable and impermeable bottom, Computers and Fluids 37(8): 10401046. http://dx.doi.org/10.1016/j.compfluid.2007.11.002

Kocabas, F.; Yildirim, N.; Donmez, S. 2010a. An artificial neural networks model for the circulation imposed on critical submergence of an intake pipe, The Kuwait Journal of Science and Engineering 37(1B): 21-34.

Kocabas, F.; Unal, S. 2010b. Compared techniques for the critical submergence of an intake in water flow, Advances in Engineering Software 41(5): 802-809. http://dx.doi.org/10.1016/j.advengsoft.2009.12.021

Lee, G. C.; Chang, S. H. 2003. Radial basis neural network function networks applied to DNBR calculation in digital core protection systems, Annals of Nuclear Energy 30(15): 1516-1572.

http://dx.doi.org/10.1016/S0306-4549(03)00099-9
Lee, J. J.; Lee, J. W.; Yi, J. H.; Yun, C. B.; Jung, H. Y. 2005. Neural networks-based damage detection for bridges considering errors in baseline finite element models, Journal of Sound and Vibration 280(3-5): 555-578. http://dx.doi.org/10.1016/j.jsv.2004.01.003

Li, Q. S. 2000. Exact solutions for free longitudinal vibrations of nonuniform rods, Journal of Sound and Vibration 234(1): 1-19. http://dx.doi.org/10.1006/jsvi.1999.2856

Li, Q. S. 2001. Free vibration analysis of non-uniform beams with an arbitrary number of cracks and concentrated masses, Journal of Sound and Vibration 252(3): 509-525. http://dx.doi.org/10.1006/jsvi.2001.4034

Liu, L.; Meng, G. 2005. Crack detection in supported beams based on neural network and support vector machine, Lecture Notes in Computer Science 3498: 597-602. http://dx.doi.org/10.1007/11427469 95

Liu, S. W.; Huang, J. H.; Sung, J. C.; Lee, C. C. 2002. Detection of cracks using neural networks and computational mechanics, Computational Methods in Applied Mechanical Engineering 191(25): 2831-2845. http://dx.doi.org/10.1016/S0045-7825(02)00221-9

Marquardt, D. 1963. An algorithm for least squares estimation of non-linear parameters, Journal of the Society for Industrial and Applied Mathematics 11(2): 431-441. http://dx.doi.org/10.1137/0111030

Marwala, T.; Hunt, H. 1999. Fault identification using finite element models and neural networks, Mechanical Systems and Signal Processing 13(3): 475-490. http://dx.doi.org/10.1006/mssp.1998.1218

Nandwana, B. P.; Maiti, S. K. 1997. Detection of the location and size of a crack in stepped cantilever beams based on measurements of natural frequencies, Journal of Sound and Vibration 203(3): 435-446. http://dx.doi.org/10.1006/jsvi.1996.0856

Nikolako poulos, P. G.; Katsareas, D. E.; Papado poulos, C. A. 1997. Crack identification in frame structures, Computers and Structures 64(1-4): 389-406. http://dx.doi.org/10.1016/S0045-7949(96)00120-4

Pandey, A. K.; Biswas, M. 1994. Damage detection in structures using changes in flexibility, Journal of Sound and Vibration 169(1): 3-17. http://dx.doi.org/10.1006/jsvi.1994.1002

Pandey, A. K.; Biswas, M.; Samman, M. M. 1991. Damage detection from changes in curvature mode shapes, Journal of Sound and Vibration 145(2): 321-332. http://dx.doi.org/10.1016/0022-460X(91)90595-B

Poggio, T.; Girosi, F. 1990. Regularization algorithms for learning that are equivalent to multilayer networks, Science 2247(4945): 978-982. http://dx.doi.org/10.1126/science.247.4945.978

Ratcliffe, C. P. 2000. A frequency and curvature based experimental method for locating damage in structures, Journal of Vibration and Acoustics-Transactions of the ASME 122(3): 324-329. http://dx.doi.org/10.1115/1.1303121

Rosales, M. B.; Filipich, C. P.; Buezas, F. S. 2009. Crack detection in beam-like structures, Engineering Structures 31(10): 2257-2264. http://dx.doi.org/10.1016/j.engstruct.2009.04.007

Saeed, R. A.; George, L. E. 2011. The use of ANN for cracks predictions in curvilinear beams based on their natural frequencies and frequency response functions, Journal of Computing 3(12): 113-125.

Sahin, M.; Shenoi, R. A. 2003. Quantification and localisation of damage in beam-like structures by using artificial neural networks with experimental validation, Engineering Structures 25(14): 1785-1802. http://dx.doi.org/10.1016/j.engstruct.2003.08.001 
Sampaio, R. P. C.; Maia, N. M. M.; Silva, J. M. M. 2003. The frequency domain assurance criterion as a tool for damage detection, Proceedings of Key Engineering Materials: Damage Assessment of Structures 245(2): 69-76. http://dx.doi.org/10.4028/www.scientific.net/KEM.245246.69

Shi, Z. Y.; Law, S. S.; Zhang L. M. 2000a. Structural damage detection from modal strain energy change, Journal of Engineering Mechanics ASCE 126(12): 1216-1223. http://dx.doi.org/10.1061/(ASCE)0733-93992000)126: $12(1216)$

Shi, Z. Y.; Law, S. S.; Zhang, L. M. 2000b. Damage localization by directly using incomplete mode shapes, Journal of Engineering Mechanics ASCE126(6): 656-660. http://dx.doi.org/10.1061/(ASCE)0733-9399(2000)126: 6(656)

Shi, Z. Y.; Law, S. S.; Zhang, L. M. 2002. Improved damage quantification from elemental modal strain energy change, Journal of Engineering Mechanics ASCE 128(5): 521-529. http://dx.doi.org/10.1061/(ASCE)0733-9399(2002)128:5(521)

Suresh, S.; Omkar, S. N.; Ganguli, R.; Mani, V. 2004. Identification of crack location and depth in a cantilever beam using a modular neural network approach, Smart Materials and Structures 13(4): 907-915. http://dx.doi.org/10.1088/0964-1726/13/4/029
Wu, D.; Law, S. S. 2004. Damage localization in plate structures from uniform load surface curvature, Journal of Sound and Vibration 276(1-2): 227-244. http://dx.doi.org/10.1016/j.jsv.2003.07.040

Wu, D.; Law, S. S. 2005. Sensitivity of uniform load surface curvature for damage identification in plate structures, Journal of Vibration and Acoustics-Transactions of the ASME 127(1): 84-92. http://dx.doi.org/10.1115/1.1857918

Wu, X.; Ghaboussi, J.; Garrett, J. H. 1992. Use of neural networks in detection of structural damage, Computers and Structures 42(4): 649-659. http://dx.doi.org/10.1016/0045-7949(92)90132-J

Yan, Y. J.; Cheng, L.; Wu, Z. Y.; Yam, L. H. 2007. Development in vibration-based structural damage detection technique, Mechanical Systems and Signal Processing 21(5): 2198-2211. http://dx.doi.org/10.1016/j.ymssp.2006.10.002

Yun, C. B.; Bahng, E. Y. 2000. Substructural identification using neural networks, Computers and Structures 77(1): 41-52. http://dx.doi.org/10.1016/S0045-7949(99)00199-6

Zapico, J. L.; Gonzalez, M. P.; Worden, K. 2003. Damage assessment using neural networks, Mechanical Systems and Signal Processing 17(1): 119-125.

http://dx.doi.org/10.1006/mssp.2002.1547

Kamil AYDIN. PhD, Associate Professor of Civil Engineering Department, Faculty of Engineering at Erciyes University, Kayseri, Turkey. He received his PhD degree from North Carolina State University, Raleigh, North Carolina, USA. He has published around 30 journal and conference papers. His areas of academic research interests include structures, structural dynamics, earthquake engineering and structural health monitoring.

Ozgur KISI. PhD, Professor of Civil Engineering Department, Faculty of Engineering at Canik Basari University, Samsun, Turkey. He received his $\mathrm{PhD}$ degree from the Istanbul Technical University, Istanbul, Turkey. He currently serves as the founding dean of Engineering Faculty. He has been awarded several times by such international institutions as International Association of Hydrological Sciences for his outstanding works. He has published more than 110 journal papers and 50 conference papers. His studies focus on the applications of new mathematical models to hydrological sciences and water resources, also called as hydro-informatics. 